\title{
Antecedentes y perspectivas de la tecnología y producción de semillas en el INIFAP ${ }^{\dagger}$
}

$\dagger$ In memoriam

del MC Juan Virgen Vargas, destacado investigador del INIFAP en tecnología de semillas

\author{
Juan José García-Rodríguez ${ }^{1}$ \\ Alfredo Josué Gámez-Vázquez ${ }^{1}$ \\ Francisco Paúl Gámez-Vázquez ${ }^{1}$ \\ María Alma Rangel-Fajardo ${ }^{2}$ \\ Andrés Mandujano-Bueno ${ }^{1}$ \\ Miguel Angel Avila-Perches ${ }^{1 \S}$
}

${ }^{1}$ Campo Experimental Bajío-INIFAP. Carretera Celaya-San Miguel de Allende km 6.5, Celaya, Guanajuato. CP. 38010. (garcia.juanjose@inifap.gob.mx; gamez.josue@inifap.gob.mx, gamez.francisco@inifap.gob.mx; mandujano.andres@inifap.gob.mx). ${ }^{2}$ Campo Experimental Mocochá-INIFAP. Antigua carretera MéridaMotul km 25, Mocochá, Yucatán, México. CP. 97454. (rangel.alma@inifap.gob.mx).

${ }^{\S}$ Autor para correspondencia: avila.miguel@inifap.gob.mx.

\section{Resumen}

En los últimos años el Instituto Nacional de Investigaciones Forestales, Agrícolas y Pecuarias ha generado tecnología para la producción de semillas. Los objetivos del presente documento es analizar los logros de la investigación científica, determinar áreas de oportunidad y colaborar en la mejora del proceso de multiplicación de semillas. Se concluyó que, con excepción de maíz, en los últimos 30 años la producción de semilla calificada en México descendió. El instituto ha generado para los principales cultivos, tecnología para multiplicar semilla de genotipos mejorados y de especies nativas, en ambos casos, la investigación en manejo postcosecha es marginal. A pesar del vínculo institucional con empresas semilleras de capital nacional, la adopción de sus semillas mejoradas ha sido reducida.

Palabras clave: aportes, evolución, manejo agronómico, oportunidad institucional.

Recibido: marzo de 2021

Aceptado: abril de 2021 
En la búsqueda de mayor rendimiento y calidad en la multiplicación de semillas se requiere información sobre las áreas de adaptación, fenología y prácticas agrícolas (Espinosa et al., 2003). Durante los últimos 35 años el Instituto Nacional de Investigaciones Forestales, Agrícolas y Pecuarias (INIFAP) ha generado tecnologías al respecto. El objetivo del presente documento es revisar los logros de la investigación científica, detectar líneas de oportunidad, satisfacer necesidades actuales de conocimiento, mayor comprensión del tema y así contribuir en la mejora del proceso de producción de semilla.

\section{Antecedentes de la producción de semillas en México}

En la primera Ley sobre Producción, Certificación y Comercio de Semillas, en 1961 fueron fijadas las bases para establecer el Sistema Nacional de Producción, Certificación y Comercio de Semilla, integrado por el Instituto Nacional de Investigaciones Agrícolas (INIA), que resultó de la fusión de la Oficina de Estudios Especiales (OEE) y el Instituto de Investigaciones Agrícolas (IIA), la Productora Nacional de Semillas (PRONASE), el Servicio Nacional de Inspección y Certificación de Semillas (SNICS), el Comité Calificador de Variedades de Plantas (CCVP) y el Registro Nacional de Variedades de Plantas (RNVP); en dicha ordenanza se mencionaba que el INIA (hoy INIFAP) era el desarrollar de variedades e híbridos y entregar la semilla original a PRONASE, quien la multiplicaría, beneficiaría y comercializaría, para ponerla a disposición de los productores agrícolas mexicanos, sustituyendo a la antigua Comisión Nacional del Maíz (Espinosa et al., 2014).

Para adecuarse a las políticas de la globalización comercial, el gobierno mexicano en la década de los 90's, efectuó cambios en su legislatura que afectaron directamente al sector agrícola, en 1991 se promulgó una nueva ley de semillas, que permitió la participación sin restricciones al sector privado en la investigación agrícola, particularmente en la producción y comercialización de semillas, además se abrió la posibilidad de que otros usuarios multiplicaran y comercializaran semilla de variedades generadas por instituciones públicas, como el INIFAP, además de PRONASE. Tras la suscripción del Tratado de Libre Comercio de América del Norte (TLCAN), México se integró a la Unión Internacional para la Protección de las Obtenciones Vegetales (UPOV) y en concordancia, en 1996 se publicó la Ley Federal de Variedades Vegetales (LFVV) con esta y la Ley de Semillas de 1991, se permitió la participación de la iniciativa privada, que aunado a políticas de inversión del sector público y presiones de organismos internacionales, originó que paulatinamente ocurriera el desmantelamiento de PRONASE (Luna et al., 2012).

En 2007, se publica la nueva Ley Federal de Producción, Certificación y Comercio de Semillas vigente, en ésta se emite la desaparición de la PRONASE, lo cual incrementó el control del comercio de semillas por unas cuantas empresas multinacionales. Esta situación propició desabasto de semillas de frijol, avena, arroz y de variedades de polinización libre de maíz en grandes superficies (Espinosa et al., 2014).

\section{Evolución de la producción de semillas en México}

En México se cultivan anualmente poco más de 18 millones de hectáreas $25 \%$ en riego y $75 \%$ en temporal. Los principales cultivos son maíz, frijol, sorgo, avena, trigo y cebada (INEGI, 2019). A nivel mundial, los incrementos observados en la producción de los principales cultivos se atribuyen en $60 \%$ al mejoramiento genético (Lee y Tollenaar, 2007). En este sentido, la semilla es el insumo de mayor importancia para incrementar el rendimiento unitario (Copeland y McDonald, 2001). 
La producción artesanal de semilla (PAS) abastece cerca de 2.7 millones de unidades de producción, generalmente en temporal y menores a 5 ha, mientras que la semilla producida por empresas abastece poco más de 0.8 millones de unidades de riego, comúnmente mayores a 5 ha, que cuentan con maquinaria y recursos ilimitados (INEGI, 2019). En México, en los últimos 30 años, la producción de semilla calificada ha fluctuado, en el periodo de 1988 a 1990 se obtuvieron cerca de 350000 t; sin embargo, para 2001 fue de poco más de 100000 t, mientras que en 2019 fue de $243197 \mathrm{t}$ (SNICS, 2021).

\section{Aportaciones del INIFAP en la tecnología de semillas}

\section{Efectos genéticos involucrados en la calidad de semilla}

En maíz en Valles Altos, Gámez (2007) señala que los efectos aditivos (a) fueron determinantes en el peso y longitud de plúmula (LP); los efectos de dominancia $(d)$ definieron la longitud de radícula (LR), y los epistáticos: $a a$ se manifestaron en la LP, los ad influyeron en la expresión de la LR y los $d d$ en la LP. Al respecto Avila (2008) indica que, en la manifestación de la calidad fisiológica, fueron importantes los efectos genéticos aditivos, no aditivos y recíprocos. En el trópico, Manjarrez et al. (2008) detectaron que la acción génica aditiva predominó en las características de vigor.

\section{Ambiente de producción}

La producción de semilla de progenitores e híbridos de maíz de Valles Altos se puede realizar en San Luis de la Paz, Guanajuato, ya que no se observaron diferencias significativas en rendimiento, peso, tamaño y calidad fisiológica de la semilla, entre localidades (Hernández, 2018). Al respecto, Espinosa y Carballo (1988a) mencionan que el mejor ambiente para incrementar semilla de líneas e híbridos de maíz adaptados a la Zona de Transición fue Francisco I. Madero, Hidalgo; no obstante, Espinosa y Carballo (1988b) indican que para H-135, la semilla certificada de mayor calidad se obtiene en localidades de El Bajío.

La producción de semilla de maíz de Valles Altos en el ciclo otoño-invierno en una localidad con ambiente cálido seco, afectó el rendimiento y vigor de plántula (Cruz et al., 1985). La cruza simple hembra del H-385A, en Tlajomulco, Jalisco, rindió 50.9\% más que en San José de Mojarras, Nayarit (Ramírez et al., 2017). En trigo de Valles Altos, el ambiente impactó la calidad de semilla (Fernández et al., 2015). En cebada en El Bajío, en la fecha de siembra del 15 de diciembre fue superior la calidad, en comparación con la del 15 de enero (Pérez et al., 2015). En esta misma región y cultivo, Arellanes (2018) señala que, la producción de semilla se debe establecer entre el 20 de noviembre y el 20 de diciembre.

De igual forma Noriega et al. (2019) en trigo señalan que la fecha de siembra del 15 de noviembre elevó la calidad física y vigor de plántula. En este mismo cultivo, del 01 al 20 de diciembre es la fecha óptima de siembra para producir semilla en el Valle de Mexicali, Baja California y San Luis Río Colorado, Sonora (Valenzuela, 2011). En Valles Altos, la fecha de siembra de principios de junio de líneas de maíz disminuyó su rendimiento y el porcentaje de semilla mediana (Virgen et $a l ., 2013)$. En este aspecto, la producción de semilla de H-40 y H-48 en Tlaxcala, se debe realizar en las zonas sur y poniente del estado (Rojas et al., 2009a; Rojas et al., 2009b). 
En El Bajío, con la semilla de frijol establecida el 5 de julio, se obtuvo mayor rendimiento; sin embargo, la calidad fisiológica fue más alta en la siembra del 5 de marzo (González et al., 2008). En esta región, Bobadilla et al. (2013) señalan que, en la siembra de avena del 31 de diciembre, se obtuvo mayor rendimiento de semilla y menor peso volumétrico.

\section{Tecnología de producción}

García et al. (2014) reportan en El Bajío, que es viable la producción de semilla del H-135 con el patrón de siembra compacto (PSC), en este aspecto, García et al. (2018) indican que los PSC 4:1 y 8:1 producen semillas de similar calidad con arreglo convencional 6:2. En cebada del Bajío, la producción de semilla en surcos a doble hilera y densidad de siembra de $100 \mathrm{~kg} \mathrm{ha}^{-1}$ mejoraron el rendimiento y calidad (Cobarrubias, 2007). En este cultivo, no se justificó aplicar 270 unidades de nitrógeno (UN) ha ${ }^{-1}$, porque no incrementó el rendimiento y calidad física (Avila et al., 2020).

En el Estado de México, aplicar 60 unidades de $\mathrm{K} \mathrm{ha}^{-1}$ en la siembra, mejoró el rendimiento y la calidad física de avena (Márquez et al., 1991), en El Bajío, utilizar $90 \mathrm{~kg}$ de semilla ha ${ }^{-1}$, incrementó el rendimiento (Bobadilla et al., 2013). En la multiplicación de semilla de líneas de sorgo, Mora et al. (1992b) señalan que, en el tratamiento sin fertilizar, el rendimiento de semilla beneficiada se elevó entre 107 y $132 \%$ al aplicar 100 o $200 \mathrm{~kg}$ de nitrógeno (N) ha ${ }^{-1}$ en la siembra respectivamente.

En El Bajío, con 75000 a 90000 plantas ha ${ }^{-1}$ y con la aplicación de $150 \mathrm{~kg} \mathrm{de} \mathrm{N}$ ha $^{-1}$ se alcanzó el mayor rendimiento de la hembra del H-374C (Cervantes et al., 2013). En esta región, se recomienda utilizar la fórmula 220-60-60, para la producción de semilla de híbridos QPM, en cambio en el Trópico Húmedo conviene aplicar 184-69-30 (Espinosa et al., 2003). En el incremento de semilla de líneas tropicales de maíz, la más alta germinación y calidad física, se presentaron con 62500 plantas ha $^{-1}$ y la dosis de fertilización 184-69-60 (Tosquy et al., 1998).

En maíz de Valles Altos, Espinosa y Tadeo (1992) mencionan que la densidad que combina alto rendimiento y calidad de semilla para ambos progenitores del H-137 es de 60000 plantas ha ${ }^{-1}$. En este entorno, Virgen et al. (2010) señalan que en Tlaxcala no hubo diferencias entre densidades de población para rendimiento, pero que con 62000 plantas ha $^{-1}$, la semilla fue de mayor tamaño y peso. Al respecto, Virgen et al. (2014) en la evaluación de líneas progenitoras de híbridos, indican que con 82500 plantas ha ${ }^{-1}$ se incrementó el rendimiento y disminuyó el peso de mil semillas.

En trigo, Valenzuela (2011) recomienda la aplicación de 210 UN en el Noroeste de México. Para la producción de frijol con fertiriego en San Luis Potosí, se recomienda aplicar el tratamiento de fertilización de 100-100-50, dosificada en el agua de riego (Jasso y Martínez, 2012). En arroz, Ortega (2014) recomienda la dosis de fertilización 184-46-30 y la aplicación foliar de fósforo, potasio y microelementos. La mayor producción de semilla de pastos forrajeros en el norte de México se obtuvo con la fertilización 120-60-00 y 60-30-00 más micorriza (Sáenz et al., 2015).

\section{Control de la floración}

En la producción de semilla híbrida de maíz, se recomiendan algunas prácticas para favorecer una buena sincronización de la floración de los progenitores:1) sembrar en dos fechas los surcos macho; 2) sembrar a una mayor profundidad un surco macho para atrasar su floración; 3)aplicación de fertilizante foliar, los productos con base en fósforo aceleran la floración y con nitrógeno, la 
atrasan; y 4) mayor densidad de población en los surcos macho para atrasar la floración (Virgen y Velásquez, 2006). En este aspecto, Torres et al. (2006) en Valles Altos, señalan que, con un corte al ras, cuando la planta tenía cuatro hojas liguladas, retrasó la floración masculina, el flameo retrasó la floración masculina y femenina. Espinosa et al. (2001) mencionan que la floración masculina de la hembra y macho del H-311, se adelantaron, al aplicar ácido giberélico. En este cultivo, diferentes dosis de fertilización al suelo no modificaron la floración masculina y femenina (Espinosa y Tadeo, 1992); no obstante, en sorgo, esta práctica si aceleró el desarrollo reproductivo (Mora et al., 1992b).

En sorgo, Garza et al. (1992) señalan que, en el progenitor adelantado, la frecuencia y láminas de riego pueden modificarse, ya que, los riegos frecuentes y pesados pueden retrasar su floración, mientras que escardas frecuentes al progenitor atrasado, la pueden adelantar, además indican que prácticas como la aplicación de 2-4D y ácido giberélico modifican la floración. En esta misma especie, la poda del follaje provocó un retraso en su fenología, por lo que permitiría sincronizar la floración entre progenitores que difieran en esa fase del desarrollo; sin embargo, debido a que hubo reducción del rendimiento de semilla, sería recomendable aplicar dicha práctica sobre el progenitor masculino (Mora et al., 1992a).

En la producción de semilla híbrida de maíz es esencial eliminar la espiga (desespigamiento) del progenitor hembra para mantener la calidad genética, en este aspecto, en Valles Altos, Espinosa y Tadeo (1998) señalan que, si se eliminan tres hojas al desespigar, no se afectó el rendimiento y la calidad física, por su parte Virgen et al. (2016a) mencionan que, en esta práctica, eliminar la hoja bandera, no disminuyó rendimiento, tamaño y peso de semilla.

\section{Manejo poscosecha}

La cebada almacenada en frascos de vidrio y plástico presentó la más alta calidad fisiológica después de 10 meses (Castillo, 2012). El silo hermético es una tecnología para la conservación de semillas, de maíz, frijol, calabaza y muchos otros más, permite evitar pérdidas por daños de plagas, hongos y roedores (García et al., 2007). Con respecto al tratamiento de maíz, Rivas et al. (2014) en Valles Altos recomiendan que se apliquen los fungicidas Thiram más Carboxin, mientras que Ramírez et al. (2019) en Jalisco, sugieren tratar al macho, B-51 y al H-391 con Metalaxil.

\section{Producción artesanal y selección de semilla de variedades criollas}

La PAS es una alternativa en regiones de poco acceso o donde el uso de semilla certificada no resulte viable por razones culturales, económicas u otras. Se ha demostrado la bondad de la PAS en frijol en Veracruz (Ugalde et al., 2014) y Zacatecas (Zandate y Galindo, 2006). Además, se ha realizado investigación para la capacitación de los productores en la selección de semilla de sus variedades criollas, en maíz, en Guanajuato (Aguirre y García, 2012) y Tlaxcala (Rojas y Arellano, 2006), así como en ajo (Reveles et al., 2009) y chile (Cabañas et al., 2006).

\section{Evaluación de la calidad de semillas}

En maíz forrajero, las variables de calidad de semilla más importantes para predecir el establecimiento en campo fueron longitud y peso de mil semillas, velocidad de emergencia y peso seco de plántula (Pérez et al., 2006). Virgen y Vargas (2001) proponen una escala de calificación del índice de velocidad de emergencia. Gámez et al. (2015) indican que la prueba fría con $8{ }^{\circ} \mathrm{C}$ permite clasificar materiales de maíz tolerantes a bajas temperaturas durante la germinación. 


\section{Vinculación del INIFAP con empresas semilleras}

La participación de empresas, principalmente trasnacionales en la industria de semillas, no elevó la producción de semilla certificada como se hubiera esperado a excepción de maíz, donde el abastecimiento de semilla se concentró en las regiones de mayor potencial productivo y en agricultores de mayores recursos económicos, pero se desatendieron las áreas de subsistencia y agricultura tradicional (Luna et al., 2012). El INIFAP recurre a fortalecer empresas de capital nacional al facilitar sus materiales mejorados y tecnología de producción, con la finalidad de reducir costos, beneficiar a las empresas semilleras locales o regionales y a productores de grano.

En los Valles Altos y Zona de Transición de México, la producción de semilla certificada de variedades mejoradas del INIFAP por empresas nacionales, es una alternativa viable para la adopción de tecnología para producir maíz. De 2005 a 2013 se vendieron 46.71 t de categoría registrada de progenitores de híbridos y variedades de maíz. Con esta semilla las empresas sembraron en promedio 260 ha para obtener categoría certificada, con una producción estimada de 1038 t por año, suficientes para sembrar anualmente 51900 ha de maíz. En este periodo, se atendieron 31 solicitudes por año. En 2005 se inició con la participación de 12 empresas hasta alcanzar un máximo de 58 en 2012, ubicadas en el Estado de México, Tlaxcala, Puebla, Morelos, Michoacán, Querétaro, Guanajuato y Jalisco (Virgen et al., 2016b). Respecto al H-520, en el Campo Experimental Cotaxtla del INIFAP, de 2008 a 2013 se vendieron 6106 y $2630 \mathrm{~kg}$ de semilla registrada de la hembra y macho, respectivamente, con lo cual las empresas establecieron 485.2 ha para la producción de la categoría certificada; en este lapso se atendieron 24 empresas ubicadas en 11 estados de México (Sierra et al., 2016).

En el Campo Experimental Centro Altos de Jalisco del INIFAP, en el periodo de 2009 a 2019, se vendieron 178.7 t de semilla registrada de progenitores de 14 híbridos de maíz, así las empresas establecieron 8932.6 ha y produjeron más de 40000 t de categoría certificada, con las que se sembraron 1.9 millones de ha para la producción comercial, las ventas se realizaron a 120 empresas ubicadas en 16 estados del país, de las cuales $80 \%$ se localizan en Jalisco, Michoacán, Nayarit y Guanajuato (Hernández, 2020).

\section{Áreas de oportunidad institucional}

Aunque el INIFAP tiene la mayor oferta de genotipos mejorados para los agricultores, estos han adoptado parcialmente solo algunos, por diferentes razones entre las que destacan: a) la institución carece de suficientes recursos humanos, económicos e infraestructura; b) adolece de reglamentos y normatividad, adecuados, c) requiere de una empresa sectorial que multiplique su semilla; d) carece de un programa de acompañamiento técnico transexenal que difunda el uso de semilla; y e) requiere el cumplimiento de las leyes de fomento al equilibrio económico del mercado, respeto a la biodiversidad así como de garantía a derechos del agricultor, investigador, productor de semilla y consumidor. Lo anterior, permitiría retomar y consolidar sus programas de investigación, multiplicación y tecnología de semillas; además de los sectoriales de desarrollo rural, que fomentan la economía y bienestar de los agricultores en nuestro país, especialmente a los de escasos recursos.

Se requiere desarrollar tecnología en las áreas de postcosecha, así como reforzar investigación de prácticas de manejo agronómico para la producción de semilla de especies de importancia económica y social. De igual forma, consolidar la investigación en el autoabastecimiento de semilla 
que realizan los pequeños y medianos agricultores. Además, fortalecer el estudio de las diferentes pruebas para evaluar calidad fisiológica, sanidad de semillas y mantenimiento varietal. Así como consolidar la evaluación de aspectos socioeconómicos que influyen en la multiplicación de semillas. Finalmente, el INIFAP debería contar con unidades para definir planes y proyectos en la multiplicación de semillas y generación de tecnología específica.

\section{Conclusiones}

En los últimos 30 años, la producción de semillas calificadas de los cultivos más importantes del país descendió, a excepción de maíz, donde las empresas trasnacionales comercializan el mayor volumen. No obstante, que el INIFAP mantiene vínculos con empresas semilleras de capital nacional, con casos de éxito documentados, el impacto es limitado. Aunque se han generado prácticas agronómicas para multiplicar semilla de genotipos mejorados y de variedades nativas de los principales cultivos, en ambos casos la investigación en manejo postcosecha es incipiente.

\section{Literatura citada}

Aguirre, G. J. A. y García, L. M. de L. 2012. Selección para el mejoramiento de maíz criollo. INIFAP. CIRCE. Campo Experimental Bajío. Celaya, Guanajuato, México. Folleto para productores núm. 4. 39 p. ISBN: 978-607-425-815-8.

Arellanes, H. O. A. 2018. La fecha de siembra y su relación con el rendimiento y calidad física de semilla de cebada. Tesis profesional. Nova Universitas. Ocotlán de Morelos, Oaxaca. 59 p.

Avila, P. M. A.; Huerta, Z. R.; Zamora, D. M. R.; Gámez, V. A. J. y López, C. M. L. 2020. Características de semilla de cebada en función de la fuente y dosis de nitrógeno. In: Saynes, S. V.; Fernández, L. F. y Ortiz, M. R. J. I. (Ed.). Uso eficiente de nitrógeno en la agricultura. México. 83-87 pp.

Avila, P. M. A. 2008. Aptitud combinatoria para vigor de semilla y caracteres agronómicos de maíz de Valles Altos. Tesis doctoral. Universidad Autónoma Agraria Antonio Narro. Buenavista, Saltillo, Coahuila, México. 120 p.

Bobadilla, M. M.; Gámez, V. A. J.; Avila, P. M. A.; García, R. J.J.; Espitia, R. E.; Moran, V. N. y Covarrubias, P. J. 2013. Rendimiento y calidad de semilla de avena en función de la fecha y densidad de siembra. Rev. Mex. Cienc. Agríc. 4(7):973-985.

Cabañas, C. B.; Galindo, G. G.; Reveles, H. M. G. y Bravo, L. A. G. 2006. Selección, producción y conservación de semilla de chile seco. In: Bravo, L. A. G.; Galindo, G. G. y Amador, R. M. D. (Comps.). Tecnología de producción de chile seco. INIFAP. CIRNOC. Campo Experimental Zacatecas. Libro técnico núm. 5. 19-44 p.

Castillo, Z. C. 2012. Almacenamiento de semilla de cebada maltera: análisis físico, proximal y deterioro fisiológico. Tesis de Maestría en Ciencias, Colegio de Postgraduados, Montecillo, Texcoco, Estado de México. 96 p.

Cervantes, O. F.; Covarrubias, P. J.; Rangel, L. J. A.; Terrón, I. A. D.; Mendoza, E. M. y Preciado, O. R. E. 2013. Densidad de población y fertilización nitrogenada en la producción de semilla híbrida de maíz. Agron. Mesoam. 24(1):101-110.

Cobarrubias, M. J. M. 2007. Comportamiento de la calidad y rendimiento en la producción de semilla de dos variedades de cebada maltera (Hordeum vulgare L.) de riego con diferentes densidades y sistemas de producción. Tesis profesional. Instituto Tecnológico de Roque. Celaya, Guanajuato. 114 p. 
Copeland, L. O. and McDonald, M. B. 2001. Principles of seed science and technology. $4^{\text {th }}(\mathrm{Ed})$. Kluwer Academic Publishers. Massachusetts, USA. 467 p.

Cruz, R. J.; Mendoza, R. M. y Carballo, C. A. 1985. Efectos del ambiente cálido seco en la producción de semilla de maíz de Valles Altos. Rev. Chapingo. 47(49):70-75.

Espinosa, C. A. y Carballo, C. A. 1988a. Adaptabilidad y rendimiento de semilla de líneas e híbridos de maíz. Agrociencia. 74(1):313-322.

Espinosa, C. A. y Carballo, C. A. 1988b. H-135 Híbrido trilineal de maíz de riego recomendado para altitudes de 1700 a 2200 metros. Rev. Fitotec. Mex. 11(2):223-224.

Espinosa, C. A. y Tadeo, R. M. 1992. Producción de semilla del híbrido doble de maíz H-137 en respuesta a la fertilización y densidad de población. Rev. Fitotec. Mex. 15(1):1-9.

Espinosa, A. y Tadeo, M. 1998. Evaluación de desespigue mecánico en híbridos dobles de maíz en los Valles Altos de México. Agron. Mesoam. 9(1):90-92.

Espinosa, A.; Tadeo, M.; Medina, H.; Gutiérrez, J. R. y Luna, L. 2001. Alternativas para favorecer la polinización y producción de semilla del híbrido H-311 de maíz. Agron. Mesoam. 12(2):229-235.

Espinosa, A.; Gómez, N.; Sierra, M.; Betanzos, E.; Caballero, F.; Coutiño, B.; Palafox, A.; Rodríguez, F.; García, A. y Cano, O. 2003. Tecnología y producción de semillas de híbridos y variedades sobresalientes de maíz de calidad proteínica (QPM) en México. Agron. Mesoam. 14(2):223-228.

Espinosa, C. A.; Turrent, F. A.; Tadeo, R. M.; San Vicente, T. A.; Gómez, M. N.; Valdivia, B. R.; Sierra, M. M. y Zamudio, G. B. 2014. Ley de semillas y ley federal de variedades vegetales y transgénicos de maíz en México. Rev. Mex. Cienc. Agríc. 5(2):293-308.

Fernández, S. R.; Carballo, C. A; Villaseñor, M. H. E. y Hernández, L. A. 2015. Calidad de la semilla de trigo de temporal en función del ambiente de producción. Rev. Mex. Cienc. Agríc. 6(6):1239-1251.

Gámez, V. A. J. 2007. Estudio genético y fisiológico de líneas y cruzas simples experimentales de maíz por su resistencia a las bajas temperaturas. Tesis doctoral. Universidad Autónoma Agraria Antonio Narro. Buenavista, Saltillo, Coahuila, México. 122 p.

Gámez, V. A. J.; De la O, O. M.; Avila, P. M. A.; Virgen, V. J.; Ruiz, T. N. A.; Gámez, V. F. P. y Ascencio, A. A. 2015. Calidad fisiológica de semilla y desarrollo de plántulas de maíz a temperaturas bajas. Rev. Mex. Cienc. Agríc. 6(8):1769-1779.

García, L. M. de L.; Aguirre, G. J. A.; Narro, S. J.; Cortés, B. E. y Rivera, R. J. G. 2007. Silo hermético para el control de plagas de granos almacenados en Guanajuato, México. Agric. Téc. Méx. 33(3):231-239.

García, R. J. J.; Ávila, P. M. A.; De la Torre, V. J. D. y Herrera, C. C. 2014. Diferentes patrones de siembra en la producción de semilla del híbrido de maíz H-135. AGROFAZ. 14(1):43-49.

García, R. J. J.; Avila, P. M. A.; Gámez, V. F. P.; De la O, O. M. y Gámez, V. A. J. 2018. Calidad física y fisiológica de semilla de maíz influenciada por el patrón de siembra de progenitores. Rev. Fitotec. Mex. Vol. 41(1):31-37.

Garza, U. E.; Céspedes, T. E. y Aguirre, A. E. 1992. Manual para la producción de semilla híbrida de sorgo en la planicie Huasteca. INIFAP. CIRNE. Campo Experimental Ébano. Ébano, S. L. P. México. Folleto técnico núm. 1. 18 p.

González, T. G.; Mendoza, H. F. M.; Covarrubias, P. J.; Morán, V. N. y Acosta, G. J. A. 2008. Rendimiento y calidad de semilla de frijol en dos épocas de siembra en la región del Bajío. Agric. Téc. Méx. 34(4):421-430. 
Hernández, V. R. 2020. Análisis de la venta de semilla registrada de maíz del 2009 al 2019. INIFAP. CIRPAC. Campo Experimental Centro Altos de Jalisco. Tepatitlán de Morelos, Jalisco, México.

Hernández, C. R. E. 2018. Estabilidad de rendimiento y calidad de semilla de progenitores de híbridos de maíz en Valles Altos de México. Tesis doctoral, Colegio de Postgraduados. Montecillo, Texcoco, Estado de México. 128 p.

INEGI. 2019. Instituto Nacional de Estadística, Geografía e Informática. Encuesta Nacional Agropecuaria. México. https://www.inegi.org.mx /temas/agricultura/.

Jasso, Ch. C. y Martínez, G. M. A. 2012. Guía para producir semilla mejorada de frijol con fertirriego en San Luis Potosí. INIFAP. CIRNE. Campo Experimental San Luis Potosí. San Luis Potosí, México. Publicación especial. 25 p.

Lee, E.A. and Tollenaar, M. 2007. Physiological basis of successful breeding strategies for maize grain yield. Crop Sci. 47(S3):S202-S215. Doi: 10.2135/cropsci2007.04.0010IPBS.

Luna, M. B. M.; Hinojosa, R. M. A.; Ayala, G. O. J.; Castillo, G. F. y Mejia, C. J. A. 2012. Perspectivas de desarrollo de la industria semillera de maíz en México. Rev. Fitotec. Mex. 35(1):1-7.

Manjarrez, S. M.; Rodríguez, H. S. A.; Gómez, M. N. O.; Moreno, M. E.; Vázquez, B. M. E.; Zamora, V. V. M. y López, B. A. 2008. Aptitud combinatoria para calidad de semilla de maíces normales y de alta calidad de proteína. Rev. Fitotec. Mex. 31(2):125-131.

Márquez, C. L. A.; Carballo, C. A.; Estrada, G. J. A. y Livera, M. M. 1991. Rendimiento y calidad de semilla de avena en relación a la fertilización y densidad de siembra. Agrociencia Serie Fitociencia: 2(3):67-81.

Mora, A. R.; Mendoza, O. L. E.; González, H. V. A. y Molina, M. J. C. 1992a. Métodos para sincronizar la floración de líneas parentales de sorgos híbridos. I. Influencia de la poda. Agrociencia. 3(4):23-35.

Mora, A. R.; Mendoza, O. L. E.; González, H. V. A. y Molina, M. J. C. 1992b. Métodos para sincronizar la floración de líneas parentales de sorgos híbridos. II. Influencia de la fertilización nitrogenada. Agrociencia. 3(4):37-51.

Noriega, C. M. A.; Cervantes, O. F.; Solís, M. E.; Andrio, E. E.; Rangel, L. J. A; Rodríguez, P. G.; Mendoza, E. M. y García, R. J. G. 2019. Efecto de la fecha de siembra sobre la calidad de semilla de trigo en El Bajío, México. Rev. Fitotec. Mex. 42(4):375-384.

Ortega, A. R. 2014. Manual para la producción de semilla de arroz. INIFAP. CIRPAC. Campo Experimental Tecomán. Tecomán, Colima, México. Folleto técnico núm. 2.53 p.

Pérez, M. C.; Hernández, L. A.; González, C. F. V.; García, de los S. G.; Carballo, C. A.; Vásquez, R. T. R. y Tovar, G. M. del R. 2006. Tamaño de semilla y relación con su calidad fisiológica en variedades de maíz para forraje. Agric. Téc. Méx. 32(3):341-352.

Pérez, R. J. A.; Mejía, C. J. A.; Zamora, D. M.; Solano, H. S. y Hernández, L. A. 2015. Evaluación de la fecha de siembra de diez genotipos de cebada maltera. Región Bajío Ciencia desde el Occidente. 2(2):18-28.

Ramírez, D. J. L.; Chuela, B. M.; Vidal, M. V. A.; Ledesma, M. A.; Alemán, de la T. I.; Salinas, M. Y.; Vallejo, D. H. L.; Ramírez, Z. R.; Gómez, M. N. O.; Ruiz, R. S.; Peña, R. A.; Reyes, M. C. A.; Vázquez, C. M. G.; Ruiz, C. J. A. y Preciado, O. R. E. 2017. H-385A. Híbrido doble modificado de maíz de grano amarillo para buen temporal y punta de riego para la zona centro occidente de México. INIFAP. CIRPAC. Campo Experimental Centro Altos de Jalisco. Tepatitlán de Morelos, Jal., México. Folleto técnico núm. 6. 34 p. 
Ramírez, D. J. L.; Alemán, de la T. I.; Ledesma, M. A.; Vidal, M. V. A.; Gómez, M. N. O.; Salinas, M. Y.; Peña, R. A.; Briones, R. D.; Ruiz, R. S.; Arias, Ch. L. E. y Bautista, R. E. 2019. H391. Híbrido trilineal de maíz de grano blanco para la zona Centro Occidente de México. INIFAP. CIRPAC. Campo Experimental Centro-Altos de Jalisco. Tepatitlán de Morelos, Jal. México. Folleto técnico núm. 3. 29 p.

Reveles, H. M.; Velásquez, V. R. y Bravo, L. A. G. 2009. Tecnología para cultivar ajo en Zacatecas. INIFAP. CIRNOC. Campo Experimental Zacatecas. Calera, Zac., México. Libro técnico núm. 11. 41-55 p.

Rivas, V. P.; Cano, S. A.; Pérez, R. P.; Zepeda, B. R. y Virgen, V. J. 2014. Fitosanidad de semillas para disminuir el impacto de pudrición de tallo y mazorca en híbridos de maíz de Valles Altos. INIFAP. CIRCE. Campo Experimental Valle de México. Desplegable técnica núm. 45.

Rojas, M. I. y Arellano, V. J. L. 2006. Selección y beneficio de semilla de variedades de maíz de polinización libre. INIFAP. CIRCE. Campo Experimental Tlaxcala. Tlaxcala, Tlax., México. Folleto técnico núm. 27. 23 p.

Rojas, M. I.; Virgen, V. J.; Velázquez, C. G. A. y Fernández, S. R. 2009a. Tecnología para la producción de semilla certificada de maíz del híbrido H-40 en Tlaxcala. INIFAP. CIRCE. Tlaxcala, Tlax., México. Folleto técnico núm. 40. 42 p.

Rojas, M. I.; Virgen, V. J.; Espinosa, C. A. y Fernández, S. R. 2009b. Tecnología para la producción de semilla certificada de maíz del híbrido H-48 en Tlaxcala. INIFAP. CIRCE. Tlaxcala, Tlax., México. Folleto técnico núm. 39. 33 p.

Sáenz, F. E.; Saucedo, T. R. A.; Morales, N. C. R.; Jurado, G. P.; Lara, M. C. R.; Melgoza, C. A. y Ortega, G. J. A. 2015.Producción y calidad de semilla de pastos forrajeros como respuesta a la fertilización en Aldama, Chihuahua. Tecnociencia Chihuahua. 9(2):111-119.

Sierra, M. M.; Rodríguez, M. F. A.; Palafox, C. A.; Espinosa, C. A.; Andrés, M. P.; Gómez, M. N. O. y Valdivia, B. R. 2016. Productividad de semilla y adopción del híbrido de maíz H-520, en el trópico de México. Agric. Soc. Des. 13(1):19-32.

SNICS. 2021. Servicio Nacional de Inspección y Certificación de Semillas. Directorio de productores, obtentores y comercializadores de semillas. Vol. 1. México. 213 p.

Torres, F. J. L.; Lewis, B. D. y Carballo, Carballo, C. A. 2006. Técnicas para sincronizar floración en la producción de semilla híbrida de maíz en los Valles Altos. In: Ortiz, T. C.; Espinosa, C. A.; Aspiroz, R. H. S. y Sahagún, C. S. (Comps.). Producción y tecnología de semillas de maíz del INIFAP para los Valles Altos y Zonas de Transición. INIFAP. CIRCE. Campo Experimental Valle de Toluca. Toluca, México. Libro técnico núm. 3. 28-32 p.

Tosquy, V. O. H.; De la Garza, G. R.; Castañón, N. G. y Morones, R. R. 1998. Fertilización edáfica y densidades de población para producción de semilla de líneas de maíz. Agric. Téc. Méx. 24(2):111-120.

Ugalde, A. F. J.; López, S. E.; Lépiz, I. R.; Viana, R. A. y Leyva, V. S. 2014. Producción artesanal de semilla de frijol con participación municipal en Veracruz, México. Agron. Mesoam. 25(1):35-44.

Valenzuela, J. A. 2011. Producción de semilla básica de trigo para el Noroeste de México. INIFAP. CIRNO. Campo Experimental Valle de Mexicali. Mexicali, B. C. México. Desplegable para productores núm. 39.2 p.

Virgen, V. J. y Vargas, R. J. M. 2001. Velocidad de emergencia, un parámetro importante para la selección por vigor de semilla de líneas e híbridos de maíz. Rev. Chapingo Ser. Ing. Agron. 4(1):49-53. 
Virgen, V. J. y Velásquez, C. G. A. 2006, Selección del terreno, aislamiento, fechas de siembra diferenciales y relaciones hembra-macho. In: Ortiz, T. C.; Espinosa, C. A.; Aspiroz, R. H. S. y Sahagún, C. S. (Comps.). Producción y tecnología de semillas de maíz del INIFAP para los Valles Altos y Zonas de Transición. INIFAP. CIRCE. Campo Experimental Valle de Toluca. Toluca, México. Libro técnico núm. 3. 22-27 pp.

Virgen, V. J.; Arellano, V. J. L.; Rojas, M. I.; Ávila P. M. A y Gutiérrez, H. G. F. 2010. Producción de semilla de cruzas simples de híbridos de maíz en Tlaxcala, México. Rev. Fitotec. Mex. 33(4):107-110.

Virgen, V. J.; Zepeda, B. R.; Arellano, V. J. L.; Avila, P. M. A. y Rojas, M. I. 2013. Producción de semilla de progenitores e híbridos de maíz de Valles Altos en dos fechas de siembra. Ciencia y Tecnol. Agrop. México. 1(1):26-32.

Virgen, V. J.; Zepeda, B. R.; Avila, P. M. A.; Espinosa, C. A.; Arellano, V. J. L. y Gámez, V. A. J. 2014. Producción de semilla de líneas progenitoras de maíz: densidad de población e interacción. Agron. Mesoam. 25(2):323-335. Doi: 10.15517/am.v25i2.15439.

Virgen, V. J.; Zepeda, B. R.; Avila, P. M. A.; Rojas, M. I.; Espinosa, C. A. y Gámez, V. A. J. 2016a. Desespigamiento en cruzas simples progenitoras de híbridos de maíz (Zea mays L.) para Valles Altos de México. Agrociencia. 50(1):43-59.

Virgen, V. J.; Zepeda, B. R.; Avila, P. M. A.; Espinosa, C. A.; Arellano, V. J. L. y Gámez, V. A. J. 2016b. Producción y calidad de semilla de maíz en Valles Altos de México. Agron. Mesoam. 27(1):191-206. Doi: 10.15517/am.v27i1.21899.

Zandate, H. R. y Galindo, G. G. 2006. Guía para la producción artesanal de semilla de frijol. INIFAP. CIRNOC. Campo Experimental Zacatecas. Calera, Zac., México. Folleto para productores núm. 33.29 p. 